\title{
Increased Early Mortality After Fludarabine and Melphalan Conditioning with Peripheral Blood Grafts in Haploidentical Hematopoietic Cell Transplantation with Post-Transplant Cyclophosphamide
}

Luke Eastburg ( $\square$ Luke_Eastburg@urmc.rochester.edu ) University of Rochester Medical Center https://orcid.org/0000-0001-6123-5848

David Russler-Germain

Washington University in St Louis School of Medicine

John DiPersio

Washington University in St Louis School of Medicine

Thomas Fountaine

University of Rochester Medical Center

Jeffrey Andolina

University of Rochester Medical Center

Ramzi Abboud

Washington University in St Louis School of Medicine

Eric Huselton

University of Rochester Medical Center

Research article

Keywords: haploidentical, hematopoietic cell transplantation, post-transplant cyclophosphamide, cytokine release syndrome, melphalan

Posted Date: April 1st, 2021

DOl: https://doi.org/10.21203/rs.3.rs-378419/v1

License: (c) (1) This work is licensed under a Creative Commons Attribution 4.0 International License. Read Full License

Version of Record: A version of this preprint was published at Leukemia \& Lymphoma on November 18th, 2021. See the published version at https://doi.org/10.1080/10428194.2021.1978087. 


\section{Abstract}

Due to the evolving use of haploidentical donor grafts in hematopoietic cell transplantation, there is increased need to better understand the risks and benefits of using bone marrow versus peripheral blood grafts, as well as how specific pre-transplantation conditioning regimens impact patient safety and treatment outcomes. We performed a retrospective analysis of 38 patients at two centers who specifically underwent haploidentical hematopoietic cell transplantation using fludarabine plus melphalan-based conditioning regimens with post-transplant cyclophosphamide and peripheral blood donor grafts. We observed an unexpectedly high rate of early non-relapse mortality of $21 \%$ at 100 days and $34 \%$ at 1 -year. In addition, $40 \%$ of all patients suffered from severe cytokine release syndrome and $45 \%$ of all patients suffered from kidney injury, often necessitating renal replacement therapy. The poor outcomes with 1year overall survival of $34 \%$, disease-free survival of $29 \%$, and non-relapse mortality of $34 \%$ motivate us to reconsider the appropriateness of the combination of fludarabine and melphalan conditioning with T-cell replete peripheral blood grafts in the setting of haploidentical hematopoietic cell transplant with posttransplant cyclophosphamide.

\section{Introduction:}

Haploidentical hematopoietic cell transplantation (haplo-HCT) is a readily available and potentially curative therapy for patients with hematological malignancies and non-malignant conditions. The use of high dose post-transplant cyclophosphamide (PTCy) with haplo-HCT with a bone marrow (BM) graft led to drastically reduced rates of graft versus host disease (GvHD) through selective depletion of highly alloreactive donor T-cells, while sparing CD34 + stem cells and regulatory T-cells ${ }^{1}$. Outcomes for haploHCT using this approach were shown to be comparable to those seen using matched unrelated donors and even matched related donors ${ }^{2}$.

Initial reports of haplo-HCT utilized a reduced-intensity conditioning (RIC) regimen with fludarabine, cyclophosphamide, and total body irradiation (Flu/Cy/TBI) ${ }^{1}$. Myeloablative conditioning (MAC) regimens were shown to reduce rates of relapse and graft failure but were associated with increased toxicity and non-relapse mortality (NRM) with other transplant types ${ }^{3}$. Increased intensity RIC regimens are often used with the aim of balancing decreased relapse risk with acceptable rates of NRM for older patients with malignant hematologic conditions. Fludarabine and melphalan (FluMel) conditioning is effective in other allogeneic transplantation settings, showing good disease-free survival with low rates of $\mathrm{NRM}^{4}$. Small studies have demonstrated the feasibility of using FluMel, with or without thiotepa, anti-thymocyte globulin (ATG), and/or low dose TBI with bone marrow grafts in the haplo-HCT plus PTCy platform ${ }^{5}$, and a number of other small, single institution, retrospective analyses have provided further evidence supporting this approach ${ }^{6}$.

Peripheral blood stem cell (PBSC) grafts have been increasingly utilized in favor of BM grafts for patients undergoing haplo-HCT due to ease of collection and reduced rates of relapse ${ }^{7}$. However, cytokine release 
syndrome (CRS) occurs in the majority of patients. While most cases of CRS are mild, $12 \%$ of patients suffer from severe CRS with hypoxic respiratory failure, end-organ dysfunction, and even death ${ }^{8}$. Most reports of haplo-HCT with PBSC grafts utilize the standard with Flu/Cy/TBI conditioning regimen. In this two-center retrospective analysis, we aim to describe the efficacy, safety, and toxicity of FluMel as a conditioning approach for haplo-HCT specifically using PBSC.

\section{Materials And Methods:}

\section{Study Design and Eligibility Criteria}

This observational, retrospective study was conducted at the University of Rochester Medical Center in Rochester, NY, and the Washington University School of Medicine in St. Louis, MO. All consecutive patients receiving a RIC regimen containing fludarabine and melphalan for haplo-HCT with PTCy for a hematologic malignancy between 2015 and 2019 were included in this analysis. Patients were not excluded on the basis of specific hematologic malignancies, prior lines of therapy, the presence or absence of active disease, or prior stem cell transplantation.

\section{Definitions and Endpoints}

We utilized the American Society for Transplantation and Cellular Therapy (ASTCT) consensus grading system for cytokine release syndrome ${ }^{9}$. Overall survival (OS) was defined as the time from day 0 of stem cell transplantation until date of death from any cause. Early mortality was defined as death prior to day +100. Disease-free survival (DFS) was defined as the time from day 0 of stem cell transplantation until date of documented evidence of relapsed disease or death from any cause, whichever occurred first. Relapse incidence (RI) was defined as the proportion of patients with documented evidence of relapsed disease, accounting for patients who died of any other cause not related to their underlying hematopoietic malignancy. Non-relapse mortality (NRM) was defined as the time from day 0 of stem cell transplantation until non-relapse death. Acute kidney injury (AKI) is defined as any of the following: increase in serum creatinine $\geq 0.3 \mathrm{mg} / \mathrm{dL}$ within 48 hours; or increase in serum creatinine $\geq 1.5 \mathrm{x}$ times baseline, which is known or presumed to have occurred within the prior 7 days; or urine volume $\leq$ $0.5 \mathrm{~mL} / \mathrm{kg} /$ hour for 6 hours.

\section{Statistical Methods}

Descriptive data are represented as medians with ranges when numerical, and as frequency and proportion when categorical. Significance testing for associations between clinical characteristics was assessed by Fisher exact testing. Survival curves for OS and DFS were calculated using the Kaplan-Meier method. RI and NRM were modeled using the competing risk analysis framework ${ }^{10-12}$. Statistical analyses were performed using R v3.6 (R Foundation for Statistical Computing, Vienna, Austria).

\section{Results:}


Thirty-eight patients with a diverse range of hematologic malignancies were included in the analysis (Table 1). The majority $(20 ; 52.6 \%)$ had acute myeloid leukemia (AML), eight $(21.1 \%)$ had myelodysplastic syndrome (MDS), and roughly $5 \%$ or fewer had primary myelofibrosis (PMF), chronic myeloid leukemia (CML), chronic myelomonocytic leukemia (CMML), multiple myeloma (MM), acute lymphoblastic leukemia (ALL), non-Hodgkin lymphoma (NHL), or Sezary Syndrome. Of the 20 patients with AML, four (20\%) had active disease, one (5\%) had measurable residual disease by AML 1-ETO RTPCR (0.001 normalized copy numbers), and the remaining $15(75 \%)$ were in complete remission at the time of transplantation. Eleven (28.9\%) of the 38 patients had undergone prior stem cell transplantation. The median age at date of transplantation was 60 years (range 20-73). The median Hematopoietic Cell Transplantation-specific Comorbidity Index ( $\mathrm{HCT}-\mathrm{Cl} ; \geq 3$ indicates high risk) score was 4 (range 0-10). Three $(7.9 \%)$ patients were male recipients with female hematopoietic cell donors. Median donor age was 41 years (range 20-71). All patients received conditioning regimens based on four doses of fludarabine at $30 \mathrm{mg} / \mathrm{m}^{2}$, and a single dose of melphalan on either day -2 or day -1 , followed by a peripheral blood haploidentical hematopoietic cell transplant with post-transplant cyclophosphamide per standard-of-care protocols. Thirty-six patients $\left(94.7 \%\right.$ ) received melphalan at $140 \mathrm{mg} / \mathrm{m}^{2}$ (two of whom also received antithymocyte globulin), and two patients (5.3\%) received melphalan at $100 \mathrm{mg} / \mathrm{m}^{2}$.

Median follow-up was 239 days (range 6-1086) at date of last analysis on March 5, 2020. As of this date of censoring, $13(34.2 \%)$ patients remained alive, and median follow-up for these surviving patients was 646 days (range 288-1086). All patients with follow-up of less than 100 days had died prior to day +100 . Median overall survival was 7.8 months ( $95 \%$ confidence interval $4.7-11.4$ months; Fig. 1A). Overall survival rates at days +100 and +365 were $76.3 \%$ and $33.5 \%$, respectively. Median disease-free survival was 6.1 months (95\% confidence interval $4.0-8.5$ months; Fig. 1B). The disease-free survival rates at days +100 and +365 were $71.1 \%$ and $28.9 \%$, respectively. With all patients mature for analysis at day + 100 , nine $(23.7 \%)$ patients suffered early mortality having died prior to day +100 . Eight $(88.9 \%)$ of these nine patients died without experiencing disease relapse. Cumulative relapse incidences at days +100 and +365 were $7.9 \%$ and $32.2 \%$, respectively (Fig. 1C). NRM rates at days +100 and +365 were $21.1 \%$ and $34.4 \%$, respectively (Fig. 1D).

Fifteen (39.5\%) of 38 patients had severe (grade 3-5) CRS. Eleven (73.3\%) of these 15 patients with severe CRS received treatment with tocilizumab. AKI occurred at a high rate in patients with severe CRS (10/15 patients, $66.7 \%)$ as compared to patients with CRS grade $0-2(7 / 23,30.4 \%, p=0.046)$. Six $(85.7 \%)$ of the seven patients from the entire cohort who required RRT had severe CRS. Early NRM was strongly associated with severe CRS, with NRM prior to day +100 occurring in seven (46.7\%) of 15 patients with severe CRS, compared to only one $(4.3 \%)$ of 23 patients with CRS grade $0-2(p=0.003)$.

Of the eight patients with early NRM, seven (87.5\%) had creatinine clearance $<90 \mathrm{~mL} / \mathrm{min}$ (by CockcroftGault formula) prior to transplantation, six $(75 \%)$ had hemoglobin $<9.5 \mathrm{~g} / \mathrm{dL}$ prior to transplantation, seven (87.5\%) developed AKI post-transplant, and four (50\%) needed renal replacement therapy (RRT) post-transplantation. This was compared to $36.6 \%, 33.3 \%, 33.3 \%$, and $10 \%$, respectively, of the patients not experiencing early NRM. The outcome of early NRM was significantly associated with each of these 
four pre- or post-transplantation clinical features $(p=0.016, p=0.050, p=0.013$, and $p=0.025)$. Early NRM was not significantly associated with age at transplantation, $\mathrm{HCT}-\mathrm{Cl}$ score, first versus second transplant, disease status at transplant, melphalan dosing, volume overload or diuretic use, or posttransplant infection.

\section{Discussion:}

Overall, we observed very poor outcomes with FluMel conditioning and PBSC grafts for haplo-HCT with PTCy. One-year OS in our cohort was $34 \%$, driven by unexpectedly high rates of NRM due to early toxicity after transplantation. Our reported NRM rates of $21 \%$ and $34 \%$ at day +100 and 1 -year, respectively, are much higher than the rates observed in other haplo-HCT studies utilizing RIC platforms with similar patient populations. A somewhat older report using FluMel conditioning with melphalan $140 \mathrm{mg} / \mathrm{m}^{2}$ on day -8 for haplo-HCT with BM grafts found NRM rates at day +100 and 1 -year to be $12 \%$ and $16 \%$, respectively ${ }^{2}$, and a subsequent cohort ${ }^{5}$ of similarly treated patients also showed a lower NRM (19\% at 1year). Besides our patients receiving PBSC grafts, our patient characteristics were comparable to both cohorts.

The high incidence of early NRM observed in our study was strongly associated with severe CRS, with $47 \%$ of these patients dying prior to day +100 . Patients with early mortality appeared to suffer from similar clinical syndromes characterized by severe CRS and/or high fevers, capillary leak, volume overload, significant edema and anasarca, weight gain, and oliguria. This was exacerbated by IV fluids administered with PTCy, often progressing to AKI, anuria, and metabolic acidosis requiring ICU admission and renal replacement therapy (RRT); some patients suffered further clinical decompensation and death. Anecdotally, while treatment with the anti-IL6R monoclonal antibody tocilizumab would rapidly ameliorate fevers, it did not appear to have an effect on patients' overall clinical course.

These poor outcomes in patients with severe CRS are consistent with previous reports. However, the incidence of severe CRS in this study was $40 \%$, approximately three-times the incidence described in previous analyses ${ }^{8,15}$. Abboud et al. reported severe CRS in $12 \%$ of patients undergoing haplo-HCT with PBSC grafts $^{8}$. Additionally, a single institution study of 146 patients undergoing haplo-HCT with PBSC

grafts reported severe CRS in $17 \%$ patients, who achieved a 6 -month OS of only $50 \%{ }^{15}$, further supporting the association between severe CRS and poor clinical outcomes. Severe CRS and early NRM were also associated with AKI and RRT requirement. Despite the excessive toxicity, this regimen did not appear particularly effective, with $33 \%$ of surviving patients relapsing.

CRS is thought to be primarily mediated by activated T-cells, and PBSC grafts contain nearly 8-fold more T-cells compared to BM grafts. Multiple reports have demonstrated the increased rates of severe CRS in patients receiving PBSC grafts compared to those receiving BM grafts for haplo-HCT with PTCy ${ }^{17}$. PBSC collection through G-CSF mobilization has been shown to be associated with different T-cell subsets than BM grafts. Different allograft T-cell subset contents have been associated with risk of acute and chronic GvHD as well as risk of relapse ${ }^{18}$. It is possible that allografts with a higher dose of alloreactive activated 
T-cells favor the development of CRS, while those with a higher number of regulatory T-cells are less likely to develop CRS. In addition to PBSC grafts, pre-transplant active disease and HLA-DRB1 mismatching have also been identified as independent predictors of grade $\geq 3 \mathrm{CRS}^{19}$. Our patient cohort, though, had a relatively low rate of active disease, as $75 \%$ of patients with AML were in remission at time of transplantation. Other factors such as degree of HLA mismatch, patient or donor age, donor sex, natural killer (NK)-killer immunoglobulin receptors haplotype, NK-ligand mismatch, ABO mismatch, or HCT-Cl have not been associated with severe CRS in prior reports $8,15,17$. However, the patients included in these prior studies almost exclusively received a Flu/Cy/TBI conditioning platform. This suggests that the high rate of severe CRS observed in our cohort may be related to the unique combination of using PBSC grafts with FluMel conditioning. One recent study found a higher rate of NRM and decreased OS in patients with $\mathrm{NHL}$ receiving FluMel conditioning for allogeneic HCT compared to fludarabine and busulfan-based conditioning ${ }^{13}$, whereas another cohort of patients with lymphoma undergoing haplo-HCT with fludarabine and cyclophosphamide conditioning did not suffer increased NRM or reduced OS ${ }^{14}$.

Melphalan is commonly used as part of fludarabine-based RIC regimens, which have been associated with decreased relapse rates and disease-free survival in other transplant types ${ }^{19}$. Melphalan pharmacokinetic studies report up to a 10 -fold interpatient variability in melphalan exposure ${ }^{20}$. Melphalan binds to proteins in the red blood cell membrane, leading to higher plasma concentrations in patients with lower hemoglobin ${ }^{21}$. Renal impairment also leads to higher concentrations as renal elimination accounts for approximately $40 \%$ of melphalan clearance. Lower hemoglobin $(<9.5 \mathrm{~g} / \mathrm{dL})$ and impaired renal function (creatinine clearance $<60 \mathrm{~mL} / \mathrm{min}$ ) were strongly associated with worse outcomes in patients who underwent FluMel conditioning and autologous hematopoietic cell transplantation, although the use of BM versus PBSC grafts was not specified in this report ${ }^{22}$. Outcomes in our patient cohort appear consistent with these findings. Theoretically, these patients may have increased melphalan exposure and resultant toxicity. Unpublished data from our group are also finding that MAC regimens are not associated with an increased risk for severe CRS, thus it is possible that melphalan has a unique toxicity profile where increased melphalan-exposure places patients at higher risk for severe CRS.

Melphalan has also been shown to have immunomodulatory effects. Treating tumor-bearing mice with melphalan results in a rapid burst of inflammatory cytokines and chemokines, as well as transient elimination of regulatory T-cells during the cellular recovery phase after melphalan-induced leukodepletion. This effect includes surges of IFN-y, IL-22, IL-5, IL-18, IL-27, CCL2, CCL3, CCL7, and $\mathrm{CXCL}^{23}$, with peak levels occurring two days after melphalan administration. This IFN-y peak would coincide with the timing of PBSC infusion in our cohort, as our patients received melphalan on either day -2 or day -1 . The combination of a melphalan-induced proinflammatory environment with T-cell replete haplo-HCT PBSC grafts may have contributed to the unacceptably high rate of severe CRS in our patients.

Our study is limited by its small sample size and retrospective nature. Patients were moderate-to-high risk and $29 \%$ had undergone prior hematopoietic cell transplantation. We also had a heterogeneous patient 
population with a diverse range of hematologic malignancies.

In conclusion, our institutions no longer use standard FluMel as conditioning for haplo-HCT with PTCy with T-cell replete PBSC grafts. Alternative regimens or variations on melphalan-based regimens, such as fractionated melphalan dosing, inclusion of $\mathrm{TBI}$, or inclusion of thiotepa, may improve outcomes but further studies are needed. There remains a significant unmet need to better understand the optimal conditioning approach for patients with aggressive hematologic malignancies undergoing haplo-HCT who are older or unfit for standard MAC, and this should be addressed in future randomized controlled trials.

\section{Declarations}

Ethical approval consent to participate: All procedures performed in studies involving human participants were in accordance with the ethical standards of the institutional and/or national research committee and with the 1964 Helsinki declaration and its later amendments or comparable ethical standards. Informed consent was obtained from all patients for being included in the study.

Consent for publication: All authors revised and approved the manuscript before final submission.

Competing interests: J.F.D. has received research funding from Incyte. L.E., D.A.R.-G., T.F., J.R.A., R.A., and E.H. have no relevant conflicts to disclose.

Funding: J.F.D. has received research funding from Incyte. L.E., D.A.R.-G., T.F., J.R.A., R.A., and E.H. have no relevant conflicts to disclose.

Authors' Contributions: L.E., D.A.R.-G., R.A., and E.H. designed research, performed research, collected data, analyzed data, and wrote the manuscript. D.A.R.-G. and E.H. performed statistical analysis. J.F.D., T.F., and J.R.A. reviewed this manuscript and assisted with data interpretation. All authors revised and approved the manuscript before final submission

Acknowledgements: R.A. is supported by the American Society of Hematology Clinical Research Training Institute, the American Society of Hematology Research Training Award for Fellows, and the National Cancer Institute of the National Institutes of Health under Award Number R25 CA190190.

\section{References}

1. Luznik L, O’Donnell PV, Symons HJ, Chen AR, Leffell MS, Zahurak M, et al. HLA-Haploidentical Bone Marrow Transplantation for Hematologic Malignancies Using Nonmyeloablative Conditioning and High-Dose, Posttransplantation Cyclophosphamide. Biol Blood Marrow Tr. 2008;14:641-50.

2. Ciurea SO, Zhang M-J, Bacigalupo AA, Bashey A, Appelbaum FR, Aljitawi OS, et al. Haploidentical transplant with posttransplant cyclophosphamide vs matched unrelated donor transplant for acute myeloid leukemia. Blood. 2015;126:1033-40. 
3. Rodriguez R, Nademanee A, Ruel N, Smith E, Krishnan A, Popplewell L, et al. Comparison of ReducedIntensity and Conventional Myeloablative Regimens for Allogeneic Transplantation in Non-Hodgkin's Lymphoma. Biol Blood Marrow Tr. 2006;12:1326-34.

4. Brammer JE, Khouri I, Gaballa S, Anderlini P, Tomuleasa C, Ahmed S, et al. Outcomes of Haploidentical Stem Cell Transplantation for Lymphoma with Melphalan-Based Conditioning. Biol Blood Marrow Tr. 2016;22:493-8.

5. Besien K van, Artz A, Champlin RE, Guarneri D, Bishop MR, Chen J, et al. Haploidentical vs haplo-cord transplant in adults under 60 years receiving fludarabine and melphalan conditioning. Blood Adv. 2019;3:1858-67.

6. Liu H, Rich ES, Godley L, Odenike O, Joseph L, Marino S, et al. Reduced-intensity conditioning with combined haploidentical and cord blood transplantation results in rapid engraftment, low GVHD, and durable remissions. Blood. 2011;118:6438-45.

7. Bashey A, Zhang M-J, McCurdy SR, Martin AS, Argall T, Anasetti C, et al. Mobilized Peripheral Blood Stem Cells Versus Unstimulated Bone Marrow As a Graft Source for T-Cell-Replete Haploidentical Donor Transplantation Using Post-Transplant Cyclophosphamide. J Clin Oncol. 2017;35:3002-9.

8. Abboud R, Keller J, Slade M, DiPersio JF, Westervelt P, Rettig MP, et al. Severe Cytokine-Release Syndrome after T Cell-Replete Peripheral Blood Haploidentical Donor Transplantation Is Associated with Poor Survival and Anti-IL-6 Therapy Is Safe and Well Tolerated. Biol Blood Marrow Tr. 2016;22:1851-60.

9. Lee DW, Santomasso BD, Locke FL, Ghobadi A, Turtle CJ, Brudno JN, et al. ASBMT Consensus Grading for Cytokine Release Syndrome and Neurological Toxicity Associated with Immune Effector Cells. Biol Blood Marrow Tr. 2018;25:625-38.

10. Scrucca L, Santucci A, Aversa F. Competing risk analysis using R: an easy guide for clinicians. Bone Marrow Transpl. 2007;40:381-7.

11. Scrucca L, Santucci A, Aversa F. Regression modeling of competing risk using R: an in depth guide for clinicians. Bone Marrow Transpl. 2010;45:1388-95.

12. Committee $O$ behalf of the ES. lacobelli $S$. Suggestions on the use of statistical methodologies in studies of the European Group for Blood and Marrow Transplantation. Bone Marrow Transpl 2013; 48: 1-37.

13. Ghosh N, Ahmed S, Ahn KW, et al. Association of Reduced-Intensity Conditioning Regimens With Overall Survival Among Patients With Non-Hodgkin Lymphoma Undergoing Allogeneic Transplant. JAMA Oncol. 2020;6(7):1011-8. doi:10.1001/jamaoncol.2020.1278.

14. Fatobene G, Rocha V, St Martin A, et al. Nonmyeloablative Alternative Donor Transplantation for Hodgkin and Non-Hodgkin Lymphoma: From the LWP-EBMT, Eurocord, and CIBMTR. J Clin Oncol. 2020;38(14):1518-26. doi:10.1200/JC0.19.02408.

15. Imus PH, Blackford AL, Bettinotti M, Luznik L, Fuchs EJ, Huff CA, et al. Severe cytokine release syndrome after haploidentical peripheral blood transplantation. Biology Blood Marrow Transplant $\mathrm{J}$ Am Soc Blood Marrow Transplant. 2019;25:2431-7. 
16. Raj RV, Hamadani M, Szabo A, Pasquini MC, Shah NN, Drobyski WR, et al. Peripheral Blood Grafts for T-Cell Replete Haploidentical Transplantation Increase the Incidence and Severity of Cytokine Release Syndrome. Biol Blood Marrow Tr. 2018;24:1664-70.

17. Vasu S, Geyer S, Bingman A, Auletta JJ, Jaglowski S, Elder P, et al. Granulocyte Colony-Stimulating Factor-Mobilized Allografts Contain Activated Immune Cell Subsets Associated with Risk of Acute and Chronic Graft-versus-Host Disease. Biol Blood Marrow Tr. 2016;22:658-68.

18. Mariotti J, Taurino D, Marino F, Bramanti S, Sarina B, Morabito L, et al. Pretransplant active disease status and HLA class II mismatching are associated with increased incidence and severity of cytokine release syndrome after haploidentical transplantation with posttransplant cyclophosphamide. Cancer Med-us. 2020;9:52-61.

19. Yamashita T, Takami A, Uchida N, Fukuda T, Eto T, Shiratori S, et al. Reduced-intensity stem cell transplantation for acute myeloid leukemia with fludarabine-based conditioning with intravenous busulfan versus melphalan. Bone Marrow Transp/2020;: 1-11.

20. Nath CE, Shaw PJ, Trotman J, Zeng L, Duffull SB, Hegarty G, et al. Population pharmacokinetics of melphalan in patients with multiple myeloma undergoing high dose therapy. Brit J Clin Pharmaco. 2010;69:484-97.

21. Shaw PJ, Nath CE, Lazarus HM. Not too little, not too much-just right! (Better ways to give high dose melphalan). Bone Marrow Transpl. 2014;49:1457-65.

22. Sweiss K, Calip GS, Johnson JJ, Rondelli D, Patel PR. Pretransplant hemoglobin and creatinine clearance correlate with treatment-free survival after autologous stem cell transplantation for multiple myeloma. Bone Marrow Transpl. 2019;54:2081-7.

23. Lu X, Ding Z-C, Cao Y, Liu C, Habtetsion T, Yu M, et al. Alkylating Agent Melphalan Augments the Efficacy of Adoptive Immunotherapy Using Tumor-Specific CD4 + T Cells. J Immunol. 2015;194:2011-21.

\section{Tables}

Table 1: Thirty-eight patients with a diverse range of hematologic malignancies were included in the analysis 


\section{Characteristic}

Total

Cohort

Total patients, number

38

Female sex, number (\% of 38 patients with data available)

$14(36.8)$

Age at transplantation, median (range)

$60(20-73)$

Donor age, median (range)

$42(20-71)$

HCT-Cl score, median (range)

$4(0-10)$

Prior allogeneic transplantation, number (\% of 38 patients with data available)

$11(28.9)$

Sex mismatch of male recipient / female donor, number (\% of 38 patients with data available)

$3(7.9)$

Diagnosis, number (\% of 38 patients with data available)

AML

$20(52.6)$

MDS

$8(21.1)$

PMF

$2(5.3)$

$\mathrm{CML}$

$1(2.6)$

CMML

$1(2.6)$

MM

$2(5.3)$

ALL

$1(2.6)$

$\mathrm{NHL}$

2 (5.3)

Sezary Syndrome

$1(2.6)$

Disease status at transplantation, number (\% of 20 patients with AML and data available)

Remission

$15(75.0)$

Active

$4(20.0)$

MRD positive

$1(5.0)$

Conditioning regimen, number (\% of 38 patients with data available)

Fludarabine + Melphalan $\left(100 \mathrm{mg} / \mathrm{m}^{2}\right)$

Fludarabine + Melphalan $\left(140 \mathrm{mg} / \mathrm{m}^{2}\right)$ 
ABO status, number (\% of 37 patients with data available)

CMV serostatus, number (\%, of 37 patients with data available)

Donor positive / Recipient positive

Donor negative / Recipient positive 7 (18.9)

Donor negative / Recipient negative $14(37.8)$

Donor positive / Recipient negative $2(5.4)$

Figures 

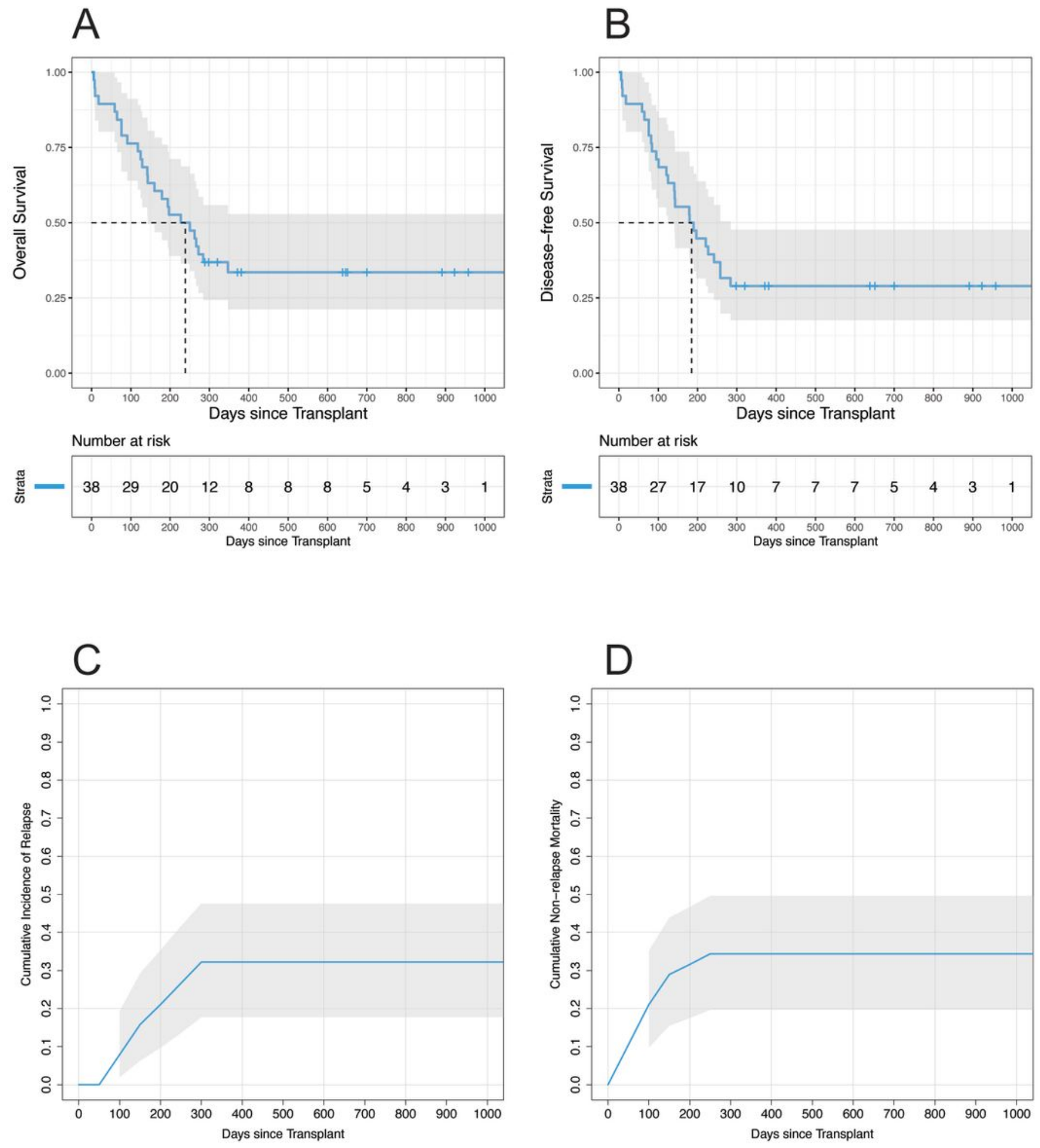

\section{Figure 1}

Median overall survival was 7.8 months ( $95 \%$ confidence interval $4.7-11.4$ months; Figure $1 \mathrm{~A}$ ). Overall survival rates at days +100 and +365 were $76.3 \%$ and $33.5 \%$, respectively. Median disease-free survival was 6.1 months (95\% confidence interval 4.0-8.5 months; Figure 1B). The disease-free survival rates at days +100 and +365 were $71.1 \%$ and $28.9 \%$, respectively. With all patients mature for analysis at day +100 , nine $(23.7 \%)$ patients suffered early mortality having died prior to day +100 . Eight $(88.9 \%)$ of these 
nine patients died without experiencing disease relapse. Cumulative relapse incidences at days +100 and +365 were $7.9 \%$ and $32.2 \%$, respectively (Figure 1C). NRM rates at days +100 and +365 were $21.1 \%$ and $34.4 \%$, respectively (Figure 1D). 